\title{
Effects of Abdominal Aortic Calcification and Facet Joint Arthritis On Lumbar Bone Mineral Density Using Dual-Energy X-Ray Absorptiometry
}

\section{Minjoon Cho}

SNU SMG Boramae Medical Center: Seoul National University Seoul Metropolitan Government Boramae Medical Center

\section{Hong Seok Kim}

Seoul National University College of Medicine

\section{Byung Sun Choi}

Seoul National University College of Medicine

\section{Jae Hyup Lee ( $\nabla$ spinelee@snu.ac.kr)}

Seoul National University College of Medicine https://orcid.org/0000-0002-2141-0266

\section{Research Article}

Keywords: Abdominal aortic calcification, osteoporosis, bone mineral density, dual-energy X-ray absorptiometry, computed tomography

Posted Date: November 3rd, 2021

DOI: https://doi.org/10.21203/rs.3.rs-1032910/v1

License: (c) (i) This work is licensed under a Creative Commons Attribution 4.0 International License. Read Full License 
1 Effects of abdominal aortic calcification and facet joint arthritis on lumbar bone

2 mineral density using dual-energy $X$-ray absorptiometry

4 Authors:

5 Min Joon Cho ${ }^{a, b}$, Hong Seok Kim ${ }^{a}$, Byung Sun Choi ${ }^{a}$, Jae Hyup Lee ${ }^{a, b}$ 6

7 Affiliations:

8 a Department of Orthopedic Surgery, Seoul National University College of Medicine, Seoul, 9 Korea

$10{ }^{\mathrm{b}}$ Department of Orthopedic Surgery, SMG-SNU Boramae Medical Center, Seoul, Korea

12 Corresponding Author:

13 Name : Jae Hyup Lee

14 ORCID ID: 0000-0002-2141-0266

15 Phone number : +(82)-10-9097-8085

16 Fax number : 02-870-3863

17 Address : Department of Orthopedic Surgery, SMG-SNU Boramae Medical Center, Seoul

18 Boramae-ro 5-gil, Dongjak-gu, Seoul, 07061 Republic of Korea

19 E-mail : spinelee@snu.ac.kr 


\section{Abstract}

Abdominal aortic calcification (AAC) may overestimate lumbar bone mineral density (BMD) examined by dual-energy X-ray absorptiometry (DXA); however, the degree of effect of AAC on lumbar BMD has not been quantified. In particular, no study has quantitatively compared and analysed segmental BMD and AAC using computed tomography (CT) scan. Thus, this study aimed to quantify the effect of AAC on BMD measurements using DXA via multiple linear regression analysis.

\section{Methods}

This study retrospectively reviewed participants $>30$ years of age who underwent DXA and spinal CT scans between 2014 and 2016. Variables that significantly affected the BMD of each lumbar segment were identified. Additionally, segmental facet joint arthritis (FJA) and AAC volume were evaluated using CT.

\section{Results}

A total of 620 subjects (153 males and 467 females) were included. The mean age was $71.6 \pm 9.1$ years (range, 31-89 years). AAC had the highest prevalence in L3 (45.2\%), followed by L4 (41.1\%). The average volume of AAC was the highest in L4 at $213.67 \pm 443.82 \mathrm{~mm}^{3}$, followed by L3 at $161.95 \pm$

$37338.09 \mathrm{~mm}^{3}$. Our regression model found that Ln (L4BMD) was significantly correlated with age, BMI, FJA, and AAC volume in female subjects. Additionally, L4 BMD might be overestimated by approximately $0.90 \%$ for every $100 \mathrm{~mm}^{3}$ increase in AAC volume. The results for $\mathrm{Ln}$ (L3BMD) were almost identical. However, these relationships were not observed in males.

\section{Conclusion}

42 According to this model, AAC may overestimate lumbar BMD examined by DXA in a dose-dependent 43 manner in females.

44 Keywords: Abdominal aortic calcification; osteoporosis; bone mineral density; dual-energy X-ray 


\section{Background}

Dual-energy X-ray absorptiometry (DXA) is a widely used diagnostic tool for osteoporosis, which can evaluate fracture risk and monitor treatment response [1]. However, confounding factors can lead to measurement errors when DXA is used to evaluate lumbar bone mineral density (BMD); these factors include vertebral size and shape variations, bone marrow fat, soft tissue calcification, and degenerative lumbar spine changes.[2,3] Among them, common degenerative changes in the elderly include facet joint arthritis (FJA) and abdominal aortic calcification (AAC) [4, 5], which have been associated with BMD. Previous studies agree that FJA overestimates BMD measurement $[6,7]$. In contrast, the effect of $\mathrm{AAC}$ on $\mathrm{BMD}$ remains controversial. One case report found that $\mathrm{AAC}$ overestimates lumbar BMD measurement on DXA due to beam path calcification [8]. On the other hand, some studies have suggested that AAC is not related to BMD [9-11]. Other studies have reported a negative correlation between AAC and BMD $[12,13]$. Based on existing literature, the impact of AAC on BMD measurements seems minimal. However, the additive effect of calcified aortic tissue on DXA measurements remain unclear. To the best of our knowledge, no previous studies have quantified AAC using computed tomography (CT) scan and conducted simultaneous evaluation of each spinal segment. Therefore, this study aimed to quantify the effect of AAC on BMD by evaluating AAC using CT scans and to examine the effect of AAC on BMD at each spine level.

\section{Study population}

This retrospective cross-sectional study reviewed subjects who underwent both DXA and Lspine CT simultaneously for various indications, including lower back pain, radiating pain, or medical consultation within 1 year at Seoul Metropolitan Government-Seoul National University, Boramae 
Medical Center between October 2014 and December 2016. In this study, subjects older than 30 years of age, which is the age where a decline in lumbar volumetric BMD is observed in both men and women, were included.[14]. Patients with the following characteristics were excluded: absence of CT scan within 1 year of DXA; history of vertebral fractures or spinal surgery involving $\geq 3$ vertebral segments between L1 and L4; history of primary or metastatic cancer involving the vertebral body; and comorbid parathyroid disease.

In this study, medical records of subjects were reviewed to determine their age, sex, weight, height, body mass index (BMI), steroid use, osteoporosis medication (BP, PTH, and SERM), and spine surgery history. Additionally, the underlying primary or metastatic cancer and parathyroid disease were evaluated.

\section{BMD measurement by DXA}

BMD was measured for all subjects using DXA, including anteroposterior lumbar DXA. For BMD evaluation, the BMD and T-score of L1, L2, L3, and L4 were examined with the total lumbar BMD and T-score. The BMD of the affected segment was excluded from the analysis if the segment had a history of surgery, such as vertebroplasty, kyphoplasty, laminectomy, and fusion surgery. A GE Lunar Prodigy Advance densitometer (General Electric, Milwaukee, WI, USA) was used to measure BMD.

\section{Evaluation by Lumbar CT}

CT and lumbar spine X-rays (if available) were analyzed within 1 year of DXA imaging. The FJA, AAC volume, fracture, and L1-L4 surgery were closely reviewed using CT by a single orthopedic surgeon who was blinded to the subjects' medical records and BMD. In addition, all measurements and evaluations using CT were recorded at each spine level to allow independent analysis of each vertebral segment. In patients with multiple CT scans within 1 year, we chose either the images obtained on a closer date to DXA, or images with fracture or surgical condition consistent with DXA. Conventional non-contrast lumbar spine CTs covering T10 to the sacrum were obtained in the supine position. A GE LightSpeed Pro 16 CT (General Electric, Milwaukee, WI, USA) was used, and images were obtained with a $2.5-\mathrm{mm}$ thickness $(120 \mathrm{kVp}, 2.5 \mathrm{pitch})$. 
FJA was evaluated in four stages from Grade 0 to Grade 3 via CT scan. Namely, grade 0 was normal; grade 1, joint space narrowing; grade 2, narrowing with sclerosis or hypertrophy; and grade 3, severe osteoarthritis with osteophyte and sclerosis.[15] For ease of analysis, the FJA grading was converted to an ordered variable from 0 to 3 , corresponding to the grade.

The AAC volume was obtained by measuring the volume of abdominal aortic calcification, which showed attenuation of $>90$ Hounsfield units on CT. Between the L1-L4 vertebra, measurements were performed for aortic calcification located anterior to each vertebra. To calculate the volume, a crosssectional area above $90 \mathrm{HU}$ was identified in the axial cut of the CT between the upper and the lower endplate of each lumbar segment and multiplied by the number of cuts of the cross-sectional area.[16] The volume of aortic calcification corresponding to each spinal segment was recorded in cubic millimeters.

\section{Vertebral compression fracture and previous spine surgery}

Vertebral compression fractures and previous spine surgeries were investigated using medical records, radiography, and CT scans. The presence of a vertebral compression fracture was evaluated and recorded for each segment. In this study, any spine surgery included fusion surgery, partial laminectomy, diskectomy, vertebroplasty, and kyphoplasty performed between L1 and L4. Fusion surgery was defined as any interbody fusion surgery or posterolateral fusion surgery of the lumbar spine that required instrumentation. The BMD of the vertebral segment with compression fracture was included in the analysis, but not BMD of vertebral segments treated with surgery.

\section{Statistical analysis}

A multiple linear regression analysis was performed to investigate the effect of abdominal aortic calcification on lumbar spine BMD. The dependent variables were the BMD of L1 to L4. Since the BMD value did not follow normality, the natural logarithm of each BMD value was obtained as the dependent variable, and normality was determined. Sex, age, BMI, FJA grading, AAC volume, vertebral fracture, fusion surgery history, osteoporosis medication, and steroid use were considered as independent 

In all enrolled study subjects, the mean BMD of women and men was $0.900 \pm 0.175$ and 1.063 $146 \pm 0.208$, respectively. The mean lumbar T-score for women and men was $-2.06( \pm 1.55)$ and $-0.87( \pm$

variables, and their significance was verified.

In our multiple linear regression model, sex was assigned a value of 0 and 1 for women and men, respectively. Age was assigned a value of years. BMI was used as a continuous variable, which was measured as $\mathrm{kg} / \mathrm{m}^{2}$ ). FJA was introduced as an ordinal scale with values ranging from 0 to 3 according to the grading system. Other factors such as the presence of vertebral fracture, fusion surgery [17], osteoporosis medication, and steroid use were assigned a value of 1 and their absence was given a value of 0 . Additionally, the independence, multicollinearity, and homoscedasticity of the standard residuals of the independent variables were examined. All data were analyzed using SPSS Statistics version 20 (IBM, Armonk, NY, USA).

\section{Results}

\section{Demographic data}

During the study period, 685 subjects were considered for the study. However, 65 patients were excluded; among them, 37 were excluded due to fractures of $>3$ vertebral segments between L1-L4, 15 underwent spinal surgery involving $\geq 3$ surgical segments between L1-L4, 12 had $\geq 3$ fractures in surgical segments between L1-L4, and one had multiple myeloma. A total of 620 subjects (153 men and 467 women) were enrolled in the analysis.

The 620 patients had a mean age of $71.6 \pm 9.1$ years (range, 31 to 89 years) and mean BMI of $24.9 \pm 4.1 \mathrm{~kg} / \mathrm{m}^{2}$. Among the 620 patients, $181(29.2 \%)$ had fractures in vertebral bodies between $\mathrm{L} 1$ and L4, $66(10.6 \%)$ had history of spine surgery, and 50 (8.1\%) had fusion surgery involving L1 through L4. (Table 1) More detailed information regarding fracture and spine surgery is provided in Supplementary Table 1.

\section{Lumbar BMD and T-score}


1.72), respectively. The mean and standard deviation of the BMD and T-score for each sex and segment are summarized in Table 2.

\section{Abdominal aortic calcification}

The overall prevalence of AAC measured by $\mathrm{CT}$ was $60.4 \%$ in women and $68.0 \%$ in men. Depending on the spinal segment, the prevalence and volume of AAC tended to increase from L1 to L4 in both men and women. Among L1 to L4, L3 (45.2\%) had the highest prevalence of AAC, followed by L4 (41.1\%), L2 (23.4\%), and L1 (11.3\%). The average AAC volume was the highest in L4 at $213.67 \pm$ $443.82 \mathrm{~mm}^{3}$, followed by $\mathrm{L} 3$ at $161.95 \pm 338.09 \mathrm{~mm}^{3}$. The mean volume of AAC was $27.39 \pm 130.27$ and $60.37 \pm 218.84 \mathrm{~mm}^{3}$ in L1 and L2, respectively, which were less than half compared to L3 or L4. (Supplementary Table 2)

In addition, AAC increased with age in both men and women, which was observed in $72.3 \%$ of the patients $>70$ years of age. Furthermore, AAC was found in $79.2 \%$ of women and $76.2 \%$ of men over 80 years of age. In contrast, only $27.7 \%$ of women and $47.6 \%$ of men aged $<60$ years demonstrated AAC. Similarly, the mean AAC volume increased gradually with age and toward the lower lumbar spine. In patients $>80$ years old, the maximum volume of AAC in L4 were $372.4 \pm 461.8 \mathrm{~mm}^{3}$ and $509.5 \pm 763.7$ $\mathrm{mm}^{3}$ in women and men, respectively. (Table 3)

\section{Facet joint arthritis}

The FJA showed different distributions between L1 and L4. FJA was the most common in L1 (81.6\%), followed by L4 (56.5\%). Grade 1 (mild FJA) was highest in L1 (48.1\%). In contrast, grade 3 (severe arthritis) was highest in L4 (15.8\%). (Figure 1)

\section{History of Osteoporosis medication and oral steroid use}

In our study population, $58(9.8 \%)$ were receiving osteoporosis drugs, and nine $(1.4 \%)$ were receiving oral steroids.( Table 1)

\section{Multiple linear regression analysis}

A normality test was performed on the BMD values of L4 from L1, which eliminated outliers. 
No BMD value satisfied the normality test. However, for the natural logarithm of BMD values from L1 to L4 (Ln (L1BMD), Ln (L2BMD), Ln (L4BMD), and Ln (L4BMD)), the Kolmogorov-Sminova value satisfied the normality $(\geq 0.05)$. Independent variables were sex, age, BMI, vertebral fracture, fusion surgery, FJA, AAC volume, osteoporosis medication, and oral steroid use.

Ln (L1BMD) and Ln (L2BMD) were correlated with sex, age, BMI, history of fusion surgery, FJA, and osteoporosis medication, but not with AAC volume (L1, $p=0.167$; L2, $p=0.154$; Table not shown). Ln (L3BMD) and Ln (L4BMD) showed statistically significant correlations with sex, age, BMI, FJA, and AAC. However, fusion surgery and osteoporosis medication were correlated with L1/L2 but not with L3 or L4. Our regression model demonstrated no significant correlation between vertebral fracture and BMD in any of the spine segments. In the multiple linear regression analysis of Ln (L3BMD) and Ln (L4BMD), the modified R-squared values were 0.326 and 0.318 , respectively. In Ln (L4BMD), regression coefficients of sex, age, BMI, FJA, and AAC volume were 0.196, -0.0066, 0.013, 0.057, and 0.0063 , respectively (all $p<0.01$ ). The results for Ln (L3BMD) were almost identical (Table 4).

\section{Multiple linear regression analysis by gender}

Multiple linear regression analysis by sex confirmed that Ln (L3BMD) and Ln (L4BMD) were positively correlated with BMI, FJA, and AAC volume and negatively correlated with age in females s. In contrast, males demonstrated no significant correlations between these factors and Ln (L3BMD), except for a positive correlation with BMI (Table 5). In Ln (L4BMD) of females, regression coefficients of age, BMI, FJA, and AAC volume were $-0.0085,0.010,0.059$, and 0.0090 , respectively (all $p<0.005)$. The results of $\mathrm{Ln}(\mathrm{L} 3 \mathrm{BMD})$ and $\mathrm{Ln}(\mathrm{L} 4 \mathrm{BMD})$ in women were almost identical, and regression coefficients of age, BMI, FJA, and AAC volume were $-0.0079,0.012,0.068$, and 0.0097 , respectively. Males demonstrated no significant correlation between age, FJA, AAC, and Ln (L4BMD). (Supplementary Table 3).

The regression model for female confirmed in this study was as follows.

$\operatorname{Ln}(\mathrm{L} 4 \mathrm{BMD})=0.236645-0.008464 *$ Age $($ year $)+0.010109 * \mathrm{BMI}\left(\mathrm{kg} / \mathrm{m}^{2}\right)+0.059080 * \mathrm{~L} 4$ FJA grade + $0.009006 *$ L4 AAC volume $\left(100 \mathrm{~mm}^{3}\right)$ 


$$
\text { L4 BMD } \propto \frac{e^{0.010109 * B M} * e^{0.059080 * F J A} * e^{0.009006 * A A C \text { volum } e}}{e^{0.008464 * A g e}}
$$
increase in BMI, approximately $6.1 \%\left(e^{0.059080}\right)$ for every increase in FJA grade, and $0.90 \%\left(e^{0.009006}\right)$ for every $100 \mathrm{~mm}^{3}$ increase in AAC volume. The measured BMD would be decreased by approximately $0.19 \%\left(1 / e^{0.008464}\right)$ annually. For example, women over 80 years of age in this study had an average L4 AAC volume of $372.4 \mathrm{~mm}^{3}$. According to our model, $e^{0.0090 \times 3.72} \fallingdotseq 1.0340$. Therefore, it can be estimated that the average L4BMD would have been overestimated by $3.4 \%$ compared to the real BMD. our final models. The Durbin-Watson values for Ln (L3BMD) and Ln (L4BMD) were 1.933 and 1.895, respectively. Thus, independence was satisfied. No multicollinearity was observed between variables.

\section{Discussion}

This study has several findings. First, AAC was common in elderly men and women $(72.3 \%$ over 70), which tended to increase with age, regardless of sex, prevalence, and volume. Second, the prevalence and volume of AAC were significantly higher at the L3-4 level compared to the L1-2 level. Third, grade 3 and grade 1 FJA were most common in L4 and L1, respectively. Fourth, females demonstrated that the BMD of L3 and L4 were significantly positively correlated with BMI, FJA, and AAC volume.

$\mathrm{AAC}$ and FJA are common degenerative changes found in the elderly population $[4,5]$ and may act as confounding factors for BMD measurements. Vascular calcification is an actively regulated process affected by the balance of several factors. It has been suggested that chronic kidney disease, diabetes mellitus, atherosclerosis, and aging potentially affect vascular calcification formation and progression. $[18,19]$ In previous studies, the prevalence and volume of AAC varied with age. Hyder et al.[20] reported 
that the prevalence was $68.4 \%$ among 6814 men and women in a MESA cohort. Vogt et al.[4] reported a prevalence of $60 \%$ and $96 \%$ for patients aged $65-69$ years and $\geq 85$ years, respectively. Another study found that the AAC volume measuring using CT was approximately $4293-6322 \mathrm{~mm}^{3}$ in subjects approximately 90 years of age.[16] Additionally, calcific deposition has been reported to begin below the abdominal aorta.[21] The results of our study appear to be consistent with previous studies in terms of prevalence, amount, and AAC location.

A $>30 \%$ prevalence of FJA has been reported in men and women in their 70 s, which increases with age.[22] One study reported that the prevalence of lumbar FJA was 37\% in non-spinal clinical indications.[23] In our study, we found a higher prevalence of FJA compared to previous studies; this may be due to the performance of the test in patients exhibiting any symptoms, rather than a symptomatic normal population. In addition, we found a relatively high prevalence of FJA in the L1, which may be attributed to the stress concentration at the thoracolumbar junction.[24] Regarding the reliability of FJA evaluation, CT has been reported to be superior to MRI.[25]

Currently, many studies have been conducted to investigate the relationship between AAC and lumbar BMD measurements using DXA. Drinka et al.[10] reported that AAC had a minor effect on BMD as evaluated by lateral X-ray. Frye et al.[12] reported that BMD is $6 \%$ higher compared to the estimation in severe AAC using a lateral X-ray study; however, the effect was minimal compared to degenerative change. Other studies insist that an independent relationship exists between AAC and osteoporosis.[18, 26]. However, these studies were all based on semi-quantitative methods of AAC-24 or AAC-8 using lateral scans of DXA or lateral lumbar X-rays. A previous study has validated these semiquantitative methods of measuring AAC severity [27]; however, they have inferior sensitivity and accuracy compared to $\mathrm{CT}$ when evaluating the volume.[28] In particular, lateral images can only detect anterior and posterior aortic wall calcifications, but not in the lateral walls. However, the accuracy of calcification using CT has been validated, even with pathology.[29]

Additionally, Kim et al.[30] used the Agatson score to quantify AAC using CT; however, they did not analyse each segment. A similar study was conducted in 2017 but also failed to compare the AAC and BMD of each segment of the lumbar spine.[16] In 2009, these limitations similarly appeared in a 

used in the treatment of osteoporosis. Comparisons between the AAC and BMD of each lumbar segment are necessary to accurately evaluate the effects of AAC. However, no previous studies have quantified AAC by comparing each segment. In this study, we attempted to overcome the limitations of previous studies by quantifying the AAC of each segment using DXA and lumbar CT captured within a year and analysing the effect on each segment's BMD.

In this study, we obtained consistent results that AAC was positively correlated with BMD measurements in L3 and L4 in women. The regression coefficients seemed to be lower than the other factors. However, in our study, $14.5 \%$ of AACs found at the L4 level in women had $>1000 \mathrm{~mm}^{3}$ in volume, with an average volume of $1615 \mathrm{~mm}^{3}$. This indicates that L4 BMC can be measured $10.7 \%$ larger on average, which is sufficient to change the T-score. Thus, in female subjects, the BMD of L3/L4 can be overestimated by AAC, especially in those older than 70 years. In contrast, the BMD of males were not associated with AAC, FJA, and age. This may be due to the lower number of males or the presence of other significant factors. Currently, no study has found the effects of AAC on the BMD of males. Therefore, further investigation of BMD in men is required. medication, and oral steroid use. The absence of relationship with fractures may be due to the comparison of BMD with fracture of a specific segment, instead of a history of osteoporotic fractures. Regarding osteoporosis medication and oral steroids, detailed distinction was not made in terms of duration, type, and dose of the drug, which can affect BMD. Nevertheless, the significant effect of AAC in L3 and L4 on lumbar BMD measurement identified in this study has important clinical implications. In particular [31], the BMD of L3 and L4 could be more significant in evaluating elderly patients with frequent osteoporotic fractures of the thoracolumbar joint than in younger people. not reflected in our model. Second, the grading of each facet joint was analysed as a continuous variable. However, the increase in one step of FJA grading may not have the same effect on BMD, which may decrease the accuracy of the quantitative effect in each factor. Nevertheless, the quantitative analysis of 


\section{Conclusion}

AAC is common regardless of sex, and demonstrates an age-dependent increase in prevalence and volume. With meticulous control of confounding factors, we found an evident correlation between $\mathrm{AAC}$ volume and BMD of L3 and L4 in females. In female subjects of old age, AAC can significantly overestimate L3 and L4 BMD using DXA in a dose-dependent manner.

\section{Declarations}

\section{Ethics approval and consent to participate}

\section{Consent for publication}

Not applicable

\section{Availability of Data and Materials}

291 The datasets generated during and/or analysed during the current study are available from the corresponding author on reasonable request.

\section{Competing interests}

294 The authors declare that they have no known competing financial interests or personal relationships that 295 could have appeared to influence the work reported in this paper.

\section{Funding}


299 MJ Cho performed data analysis and manuscript writing. HS Kim and BS Choi performed data collection 300 and literature review. JH Lee participated in the study design and manuscript proofreading. All authors 301 read and approved the final manuscript.

\section{Acknowledgements}

303 I am deeply grateful for Ji-Ho, Lee for his academic advice.

\section{List of Abbreviations}

306 AAC - abdominal aortic calcification

307 BMD - bone mineral density

308 BMI - body mass index

309 CT - computed tomography

310 DXA - dual-energy x-ray absorptiometry

311 FJA - facet joint arthritis 


\section{References}

314 1. Blake GM, Fogelman I (2007) Role of dual-energy X-ray absorptiometry in the diagnosis and treatment of osteoporosis. Journal of Clinical Densitometry 10:102-110

2. Krølner B, Berthelsen B, Nielsen SP (1982) Assessment of vertebral osteopenia: Diagnosis 23:517-521

3. Ross PD, Wasnich RD, Vogel JM (1988) Detection of prefracture spinal osteoporosis using bone mineral absorptiometry. Journal of Bone and Mineral Research 3:1-11

4. Vogt MT, Valentin RS, Forrest KYZ, Nevitt MC, Cauley JA (1997) Bone mineral density

5. Ko S, Vaccaro AR, Lee S, Lee J, Chang H (2014) The prevalence of lumbar spine facet the rheumatic diseases 65:234-238 (1997) Impact of spinal degenerative changes on the evaluation of bone mineral 
8. Smith J-A, Vento JA, Spencer RP, Tendler BE (1999) Aortic calcification contributing to bone densitometry measurement. Journal of Clinical Densitometry 2:181-183

9. Masud T, Langley S, Wiltshire P, Doyle D, Spector T (1993) Effect of spinal osteophytosis on bone mineral density measurements in vertebral osteoporosis. BMJ: British Medical Journal 307:172

10. Drinka PJ, DeSmet AA, Bauwens SF, Rogot A (1992) The effect of overlying calcification on lumbar bone densitometry. Calcified tissue international 50:507-510

11. Drinka P, Bauwens S, DeSmet A (1992) Lack of correlation between aortic

12. Frye MA, Melton III LJ, Bryant SC, Fitzpatrick LA, Wahner HW, Schwartz RS, Riggs BL (1992) Osteoporosis and calcification of the aorta. Bone and mineral 19:185-194

13. Schulz E, Arfai K, Liu X, Sayre J, Gilsanz V (2004) Aortic calcification and the risk of 89:4246-4253 PA, Khosla S (2008) A population-based assessment of rates of bone loss at multiple skeletal sites: evidence for substantial trabecular bone loss in young adult women 
and men. Journal of Bone and Mineral Research 23:205-214

15. Pathria M, Sartoris D, Resnick D (1987) Osteoarthritis of the facet joints: accuracy of oblique radiographic assessment. Radiology 164:227-230

16. Idoate F, Cadore EL, Casas-Herrero A, Zambom-Ferraresi F, Martínez-Velilla N,

18. Flipon E, Liabeuf S, Fardellone P, Mentaverri R, Ryckelynck T, Grados F, Kamel S,

17. Akazawa T, Kotani T, Sakuma T, Katogi T, Minami S, Niki H, Torii Y, Morioka S, Orita S, Inage K (2017) Bone mineral density and physical performance of female patients 27 years or longer after surgery for adolescent idiopathic scoliosis. Asian spine journal $11: 780$ Massy Z, Dargent-Molina P, Brazier M (2012) Is vascular calcification associated with bone mineral density and osteoporotic fractures in ambulatory, elderly women? Osteoporosis International 23:1533-1539

19. Johnson RC, Leopold JA, Loscalzo J (2006) Vascular calcification: pathobiological mechanisms and clinical implications. Circulation research 99:1044-1059 JJ, Criqui MH (2008) Association of coronary artery and aortic calcium with lumbar 
21. Kauppila LI, Polak JF, Cupples LA, Hannan MT, Kiel DP, Wilson PW (1997) New indices to classify location, severity and progression of calcific lesions in the abdominal aorta: a 25-year follow-up study. Atherosclerosis 132:245-250 asymptomatic cervical and lumbar facet arthropathy: a computed tomography study. Asian spine journal 13:417

24. Brant-Zawadzki M, Jeffrey Jr RB, Minagi H, Pitts LH (1982) High resolution CT of thoracolumbar fractures. American journal of roentgenology 138:699-704

25. Berg L, Thoresen H, Neckelmann G, Furunes H, Hellum C, Espeland A (2019) Facet arthropathy evaluation: CT or MRI? European radiology 29:4990-4998

26. Simon S-p, Fodor D, Muntean L, Poanta L, Cristea P, Rednic S (2014) Bone mineral density, vertebral fractures and body mass index in postmenopausal women with abdominal aortic calcification. Endocrine research 39:1-6

27. Toussaint ND, Lau KK, Strauss BJ, Polkinghorne KR, Kerr PG (2009) Determination and 
validation of aortic calcification measurement from lateral bone densitometry in dialysis patients. Clinical Journal of the American Society of Nephrology 4:119-127

28. Agatston AS, Janowitz WR, Hildner FJ, Zusmer NR, Viamonte M, Detrano R (1990) Quantification of coronary artery calcium using ultrafast computed tomography. Journal of the American College of Cardiology 15:827-832

29. Rumberger JA, Schwartz RS, Simons DB, Sheedy PF, Edwards WD, Fitzpatrick LA (1994) Relation of coronary calcium determined by electron beam computed tomography and lumen narrowing determined by autopsy. American Journal of Cardiology 73:1169-1173

30. Kim KJ, Kim KM, Park KH, Choi HS, Rhee Y, Lee YH, Cha BS, Kim MJ, Oh SM, Brown JK (2012) Aortic calcification and bone metabolism: the relationship between aortic calcification, BMD, vertebral fracture, 25-hydroxyvitamin D, and osteocalcin. Calcified tissue international 91:370-378

31. Cho MJ, Moon S-H, Lee JH, Lee J-H (2021) Association between Osteoporotic Vertebral Compression Fractures and Age, Bone Mineral Density, and European Quality of Life-5 Dimensions in Korean Postmenopausal Women: A Nationwide Cross-sectional Observational Study. Clinics in orthopedic surgery 13:207 
408 Table \& Figure Legends

409 Table 1. Demographic data of subjects

410 Table 2. Lumbar BMD and T-score of study population

411 Table 3. Abdominal aortic calcification anterior to each lumbar vertebra with respect to

412 age group and gender

413 Table 4. Linear regression analysis of Ln (L3BMD) \& Ln (L4 BMD)

414 Table 5. Linear regression analysis of Ln (L4 BMD) of female and male subjects

415

$416 \quad$ Figure Legend

417 Figure 1. Facet joint arthritis from L1 to L4

418

419 Supplementary Table 1. Detailed information of fracture and spine surgery.

420 Supplementary Table 2. Abdominal aortic calcification with respect to vertebral level 421 and gender

422 Supplementary Table 3. Linear regression analysis of Ln (L3 BMD) in female and male 423 subjects 
425 Table 1. Demographic data of subjects

\begin{tabular}{lccc}
\hline & Female $(\mathrm{n}=467)$ & Male $(\mathrm{n}=153)$ & Total(n=620) \\
\cline { 2 - 4 } Age & $71.64( \pm 8.77)$ & $71.42( \pm 10.09)$ & $71.58( \pm 9.11)$ \\
BMI $\left(\mathrm{kg} / \mathrm{m}^{2}\right)$ & $25.42( \pm 4.20)$ & $23.46( \pm 3.51)$ & $24.93( \pm 4.13)$ \\
L-BMD average & $0.900( \pm 0.175)$ & $1.063( \pm 0.208)$ & $0.940( \pm 0.196)$ \\
Any vertebral fracture between L1-L4 & $133(28.5 \%)$ & $48(31.4 \%)$ & $181(29.2 \%)$ \\
Any spine surgery between L1-L4* & $55(11.8 \%)$ & $11(7.2 \%)$ & $66(10.6 \%)$ \\
Fusion surgery between L1-L4** & $40(8.6 \%)$ & $10(6.5 \%)$ & $50(8.1 \%)$ \\
Osteoporosis med & $55(11.80 \%)$ & $3(2.00 \%)$ & $58(9.3 \%)$ \\
$\quad$ Bisphosphonate & $35(7.50 \%)$ & $2(1.30 \%)$ & $37(5.9 \%)$ \\
\multicolumn{1}{c}{ SERM } & $16(3.40 \%)$ & $2(1.30 \%)$ & $18(2.9 \%)$ \\
Oral steroid usage & $8(1.70 \%)$ & $1(0.70 \%)$ & $9(1.4 \%)$ \\
\hline
\end{tabular}

426 *Any spine surgery includes interbody fusion, posterolateral fusion, partial laminectomy, diskectomy, vertebroplasty,

427 and kyphoplasty.

$428 * *$ Fusion surgery includes any interbody fusion and posterolateral fusion which needs instrumentation. 
430 Table 2. Lumbar BMD and T-score of study population

\begin{tabular}{cccc}
\hline & Female $(\mathrm{n}=467)$ & Male $(\mathrm{n}=153)$ & Total $(\mathrm{n}=620)$ \\
\cline { 2 - 4 } L1 BMD & $0.823( \pm 0.213)$ & $0.981( \pm 0.209)$ & $0.861( \pm 0.225)$ \\
L2 BMD & $0.879( \pm 0.228)$ & $1.076( \pm 0.218)$ & $0.926( \pm 0.241)$ \\
L3 BMD & $0.907( \pm 0.364)$ & $1.117( \pm 0.283)$ & $0.957( \pm 0.357)$ \\
L4 BMD & $0.812( \pm 0.624)$ & $1.074( \pm 0.517)$ & $0.874( \pm 0.610)$ \\
Lumbar BMD average & $0.900( \pm 0.175)$ & $1.063( \pm 0.208)$ & $0.940( \pm 0.196)$ \\
\hline L1 T-score & $-2.02( \pm 1.36)$ & $-1.06( \pm 1.71)$ & $-1.79( \pm 1.51)$ \\
L2 T-score & $-2.11( \pm 2.18)$ & $-0.97( \pm 1.83)$ & $-1.84( \pm 2.16)$ \\
L3 T-score & $-2.12( \pm 1.79)$ & $-0.61( \pm 1.90)$ & $-1.78( \pm 1.93)$ \\
L4 T-score & $-1.76( \pm 1.74)$ & $-0.51( \pm 1.95)$ & $-1.47( \pm 1.87)$ \\
Lumbar T-score Average & $-2.06( \pm 1.55)$ & $-0.87( \pm 1.72)$ & $-1.76( \pm 1.67)$ \\
\hline Means and standard deviations of segmental BMD and T-score. & &
\end{tabular}

431 Means and standard deviations of segmental BMD and T-score. 
Table 3. Abdominal aortic calcification anterior to each lumbar vertebra with respect to age group and gender

\begin{tabular}{|c|c|c|c|c|c|c|c|c|c|}
\hline \multirow{8}{*}{ Female } & & \multicolumn{8}{|c|}{ Age(years) } \\
\hline & & \multicolumn{2}{|c|}{$\leq 60(\mathrm{n}=47)$} & \multicolumn{2}{|c|}{$61-70(n=145)$} & \multicolumn{2}{|c|}{$71-80(n=203)$} & \multicolumn{2}{|c|}{$>80(\mathrm{n}=72)$} \\
\hline & & $\mathrm{N}(\%)$ & AC volume* & $\mathrm{N}(\%)$ & AC volume* & $\mathrm{N}(\%)$ & AC volume* & $\mathrm{N}(\%)$ & AC volume* \\
\hline & L1 AAC & $1(2.1)$ & $0.9( \pm 5.9)$ & $9(6.2)$ & $9.8( \pm 68.0)$ & $33(16.3)$ & $35.4( \pm 108.0)$ & $12(16.7)$ & $41.9( \pm 126.3)$ \\
\hline & L2 AAC & $2(4.3)$ & $2.3( \pm 11.3)$ & $17(11.7)$ & $20.8( \pm 120.1)$ & $53(26.1)$ & $48.6( \pm 127.4)$ & $28(38.9)$ & $98.8( \pm 246.2)$ \\
\hline & L3 AAC & $9(19.1)$ & $21.6( \pm 79.2)$ & $45(31.0)$ & $80.3( \pm 222.1)$ & 101(49.8) & $168.1( \pm 303.2)$ & $47(65.3)$ & $264.0( \pm 333.7)$ \\
\hline & L4 AAC & $4(8.5)$ & $8.6( \pm 34.1)$ & $43(29.7)$ & $89.7( \pm 229.4)$ & $98(48.3)$ & $231.2( \pm 458.6)$ & $43(59.7)$ & $372.4( \pm 461.8)$ \\
\hline & \multirow[t]{2}{*}{ Total } & \multicolumn{2}{|l|}{$13(27.7)$} & \multicolumn{2}{|l|}{$72(49.7)$} & \multicolumn{2}{|l|}{$140(69.0)$} & \multicolumn{2}{|l|}{$57(79.2)$} \\
\hline \multirow{5}{*}{ Male } & & \multicolumn{2}{|c|}{$\leq 60(\mathrm{n}=21)$} & \multicolumn{2}{|c|}{$61-70(n=31)$} & \multicolumn{2}{|c|}{$71-80(n=80)$} & \multicolumn{2}{|c|}{$>80(\mathrm{n}=21)$} \\
\hline & L1 AAC & $1(4.8)$ & $0.4( \pm 1.9)$ & $3(9.7)$ & $21.4( \pm 90.1)$ & $7(8.8)$ & $23.6( \pm 99.9)$ & $4(19.1)$ & $131.3( \pm 498.3)$ \\
\hline & L2 AAC & $4(19.0)$ & $17.0( \pm 42.1)$ & $6(19.4)$ & $67.7( \pm 179.3)$ & $26(32.5)$ & $116.4( \pm 277.6)$ & $9(42.9)$ & $265.6( \pm 748.2)$ \\
\hline & L3 AAC & $9(42.9)$ & $63.5( \pm 104.3)$ & $14(45.2)$ & $167.5( \pm 427.5)$ & $43(53.8)$ & $260.1( \pm 521.1)$ & $12(57.1)$ & $347.4( \pm 519.5)$ \\
\hline & L4 AAC & $4(19.0)$ & $136.8( \pm 400.2)$ & $11(35.5)$ & $196.3( \pm 403.3)$ & $38(47.5)$ & $320.9( \pm 594.7)$ & $14(66.7)$ & $509.5( \pm 763.7)$ \\
\hline
\end{tabular}


Table 4. Linear regression analysis of Ln (L3BMD) \& Ln (L4 BMD)

\begin{tabular}{|c|c|c|c|c|c|c|}
\hline & Independent variable & $\mathrm{B}$ & $\mathrm{SE}$ & Beta & $\mathrm{p}$ & VIF \\
\hline \multirow{6}{*}{ (L3BMD) } & Constant & -0.040 & .081 & & .622 & \\
\hline & Gender $($ female $=0$, male $=1)$ & 0.188 & .018 & .376 & .000 & 1.063 \\
\hline & Age(years) & -0.0062 & .001 & -.266 & .000 & 1.074 \\
\hline & $\operatorname{BMI}\left(\mathrm{kg} / \mathrm{m}^{2}\right)^{*}$ & 0.014 & .002 & .259 & .000 & 1.060 \\
\hline & Grade of Facet joint arthritis(0-3) & 0.060 & .008 & .272 & .000 & 1.016 \\
\hline & Volume of AAC $\left(100 \mathrm{~mm}^{3}\right)$ & 0.0065 & .000 & .102 & .005 & 1.063 \\
\hline \multirow{6}{*}{ (L3BMD) } & Constant & 0.030 & .087 & & .730 & \\
\hline & Gender $($ female $=0$, male $=1)$ & 0.196 & .019 & .384 & .000 & 1.072 \\
\hline & Age(years) & -0.0066 & .001 & -.270 & .000 & 1.088 \\
\hline & $\operatorname{BMI}\left(\mathrm{kg} / \mathrm{m}^{2}\right)^{*}$ & 0.013 & .002 & .254 & .000 & 1.065 \\
\hline & Grade of Facet joint arthritis(0-3) & 0.057 & .008 & .284 & .000 & 1.031 \\
\hline & Volume of AAC $\left(100 \mathrm{~mm}^{3}\right)$ & 0.0063 & .000 & .117 & .002 & 1.072 \\
\hline
\end{tabular}

SE stands for standard error

VIF stands for Variance Inflation Factor. 
Table 5 Linear regression analysis of Ln (L4 BMD) of female \& male subjects

\begin{tabular}{|c|c|c|c|c|c|c|}
\hline & Independent variable & $\mathrm{B}$ & $\mathrm{SE}$ & beta & $\mathrm{p}$ value & VIF \\
\hline \multirow{5}{*}{ Female } & Constant & 0.237 & .098 & & .016 & \\
\hline & Age(years) & -0.0085 & .001 & -.355 & .000 & 1.097 \\
\hline & $\mathrm{BMI}(\mathrm{kg} / \mathrm{m} 2)^{*}$ & 0.010 & .002 & .213 & .000 & 1.010 \\
\hline & Grade of Facet joint arthritis $(0-3)$ & 0.059 & .008 & .318 & .000 & 1.006 \\
\hline & Volume of AAC (100mm3) & 0.0090 & .000 & .155 & .001 & 1.087 \\
\hline \multirow{5}{*}{ Male } & Constant & -0.425 & .178 & & .018 & \\
\hline & Age(years) & -0.0019 & .002 & -.089 & .309 & 1.190 \\
\hline & $\mathrm{BMI}(\mathrm{kg} / \mathrm{m} 2)^{*}$ & 0.028 & .005 & .455 & .000 & 1.035 \\
\hline & Grade of Facet joint arthritis $(0-3)$ & 0.033 & .018 & .161 & .064 & 1.174 \\
\hline & Volume of AAC (100mm3) & 0.0044 & .000 & 109 & .183 & 1.044 \\
\hline
\end{tabular}

VIF stands for Variance Inflation Factor. 
Supplement data

Supplementary Table 1. Detailed information of fracture and spine surgery.

\begin{tabular}{lccc}
\hline & Female(n=467) & Male(n=153) & Total(n=620) \\
\cline { 2 - 4 } Any spine fracture between L1-L4 & $133(28.5 \%)$ & $48(31.4 \%)$ & $181(29.2 \%)$ \\
Fracture (L1 : L2 : L3 : L4 ) & $66(14.1 \%): 35(7.5 \%): 27(5.8 \%): 1$ & $23(15.0 \%): 15(9.8 \%): 8(5.2 \%):$ & $89(14.4 \%): 50(8.1 \%): 35(5.6 \%): 2$ \\
& $2(2.6 \%)$ & $8(5.2 \%)$ & $0(3.2 \%)$ \\
Any spine surgery between L1- & $55(11.8 \%)$ & $11(7.2 \%)$ & $66(10.6 \%)$ \\
L4* & $40(8.6 \%)$ & $10(6.5 \%)$ & $50(8.1 \%)$ \\
Fusion surgery between L1-L4** & $4(1.9 \%): 3(0.6 \%): 14(3.0 \%): 44(9$. & $1(0.7 \%): 1(0.7 \%): 2(1.3 \%): 9(5$. & $10(1.6 \%): 4(0.6 \%): 16(2.6 \%): 53($ \\
Operation level (L1 : L2 : L3 : L4 ) & $4 \%)$ & $9 \%)$ & $8.5 \%)$ \\
\hline *Any spine surgery includes interbody fusion, posterolateral fusion, partial laminectomy, diskectomy, vertebroplasty, and kyphoplasty.
\end{tabular}

**Fusion surgery includes any interbody fusion and posterolateral fusion which needs instrumentation. 
Supplementary Table 2. Abdominal aortic calcification with respect to vertebral level and gender

\begin{tabular}{cccc}
\hline & Female $(\mathrm{n}=467)$ & Male $(\mathrm{n}=153)$ & Total $(\mathrm{n}=620)$ \\
\cline { 2 - 4 } AAC between L1-L4 & $282(60.4 \%)$ & $104(68.0 \%)$ & $386(62.3 \%)$ \\
\hline AAC of L1* & $55(11.8 \%)$ & $15(9.8 \%)$ & $70(11.3 \%)$ \\
Volume $\left(\mathrm{mm}^{3}\right)$ & $24.98( \pm 95.62)$ & $34.75( \pm 202.51)$ & $27.39( \pm 130.27)$ \\
AAC of L2* & $100(21.4 \%)$ & $45(29.4 \%)$ & $145(23.4 \%)$ \\
Volume $\left(\mathrm{mm}^{3}\right)$ & $43.02( \pm 146.83)$ & $113.34( \pm 353.84)$ & $60.37( \pm 218.84)$ \\
AAC of L3* & $202(43.3 \%)$ & $78(51.0 \%)$ & $280(45.2 \%)$ \\
Volume $\left(\mathrm{mm}^{3}\right)$ & $140.86( \pm 279.12)$ & $226.32( \pm 470.24)$ & $161.95( \pm 338.09)$ \\
AAC of L4* & $188(40.3 \%)$ & $67(43.8 \%)$ & $255(41.1 \%)$ \\
Volume $\left(\mathrm{mm}^{3}\right)$ & $186.61( \pm 390.35)$ & $296.29( \pm 570.88)$ & $213.67( \pm 443.82)$ \\
\hline
\end{tabular}

*Number of subjects and prevalence 
Supplementary Table 3

A. Linear regression analysis of Ln (L3 BMD) of female subjects

\begin{tabular}{|c|c|c|c|c|c|}
\hline Independent variable & B & $\mathrm{SE}$ & beta & $\mathrm{p}$ value & VIF \\
\hline Constant & 0.133 & .094 & & .158 & \\
\hline Age(years) & -0.0079 & .001 & -.342 & .000 & 1.078 \\
\hline $\operatorname{BMI}\left(\mathrm{kg} / \mathrm{m}^{2}\right)^{*}$ & 0.012 & .002 & .225 & .000 & 1.021 \\
\hline Grade of Facet joint arthritis $(0-3)$ & 0.068 & .009 & .319 & .000 & 1.008 \\
\hline Volume of AAC $\left(100 \mathrm{~mm}^{3}\right)$ & 0.0097 & .000 & .130 & .002 & 1.059 \\
\hline
\end{tabular}

B. Linear regression analysis of Ln (L3 BMD) of male subjects

\begin{tabular}{|c|c|c|c|c|c|}
\hline Independent variable & $\mathrm{B}$ & SE & beta & $\mathrm{p}$ value & VIF \\
\hline Constant & -0.282 & .154 & & .018 & \\
\hline Age(years) & -0.0023 & .002 & .069 & .309 & 1.115 \\
\hline $\operatorname{BMI}\left(\mathrm{kg} / \mathrm{m}^{2}\right)^{*}$ & 0.022 & .004 & .156 & .000 & 1.004 \\
\hline Grade of Facet joint arthritis $(0-3)$ & 0.033 & .016 & .000 & .064 & 1.075 \\
\hline Volume of AAC $\left(100 \mathrm{~mm}^{3}\right)$ & 0.000037 & .000 & .036 & .183 & 1.049 \\
\hline
\end{tabular}


Figures

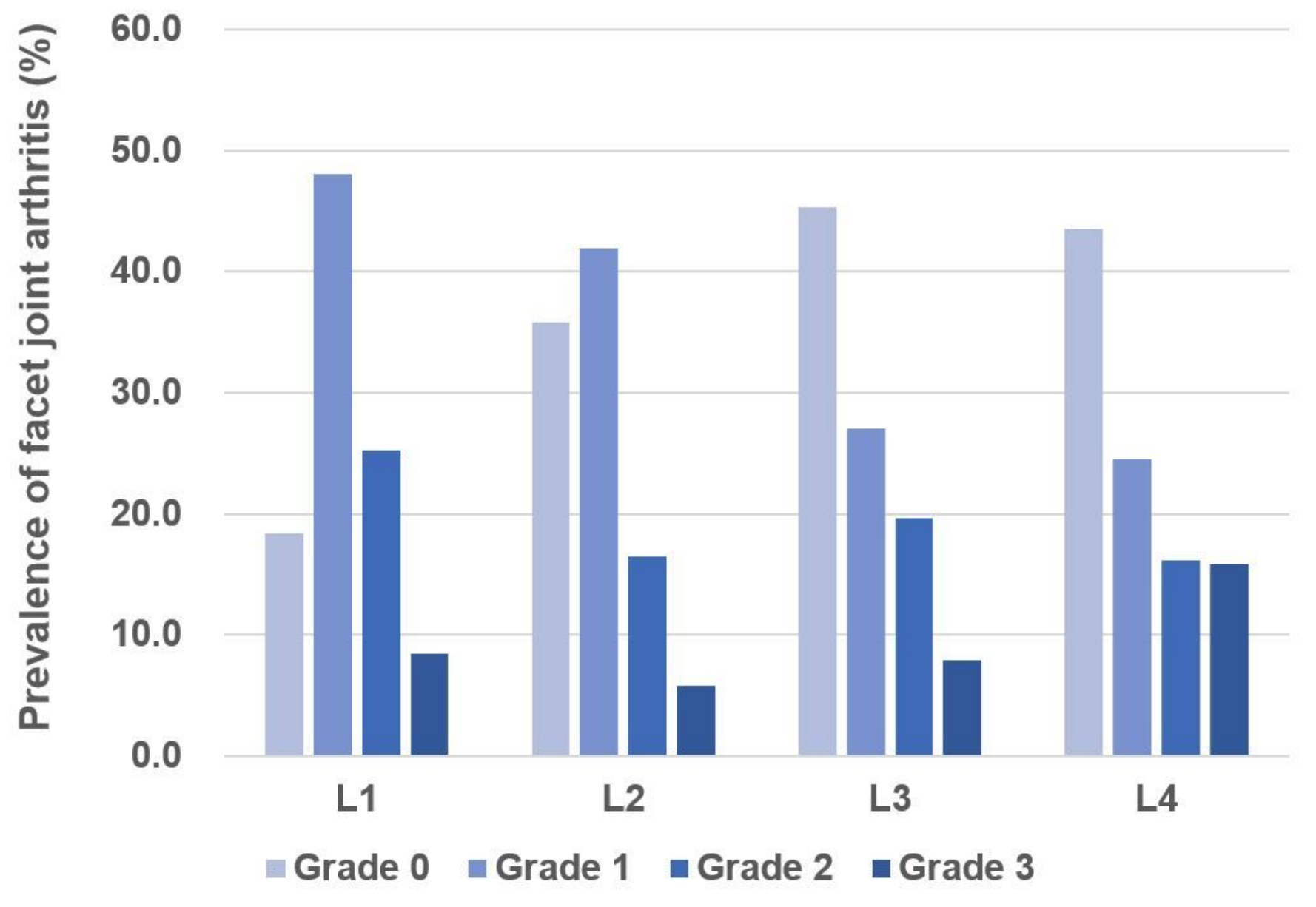

Figure 1

Facet joint arthritis from L1 to L4 